Proceedings of International Mathematical Sciences

ISSN:2717-6355, URL:HTTPS://DERGIPARK.ORG.TR/TR/PUB/PIMS

Volume II Issue 2 (2020), Pages 96-102. Doi: 10.47086/PIms.689633

\title{
CHARACTERIZATION OF ABSOLUTELY NORM ATTAINING COMPACT HYPONORMAL OPERATORS
}

\author{
BENARD OKELO \\ ODINGA UNIVERSITY OF SCIENCE AND TECHNOLOGY, BONDO, KENYA. \\ ORCID NUMBER:0000-0003-3963-1910
}

\begin{abstract}
General characterization of Hilbert space operators has been a subject of interest to many mathematicians for decades. In this paper, we characterize absolute norm-attainability for compact hyponormal operators. We give necessary and sufficient conditions for a bounded linear compact hyponormal operator on an infinite dimensional complex Hilbert space to be absolutely norm attaining. Moreover, we discuss the structure of compact hyponormal operators when they are self-adjoint and normal. Lastly, we discuss in general, other properties of compact hyponormal operators when they are absolutely norm attaining and their commutators.
\end{abstract}

\section{INTRODUCTION}

The study of norm attaining operators has been interesting to many mathematicians and researchers over decades( see [1, 2] and [5]). The class of absolutely norm attaining operators between complex Hilbert spaces was introduced by [1] and they discussed several important examples and properties of these operators. The class of absolutely norm attaining operators is denoted by $\mathcal{A N}(H)$. A synonymous class called norm-attainable operators have also been discussed by Okelo in 4 and it has been determined that they share similar characteristics. In this paper, we give necessary and sufficient conditions for an operator to be hyponormal and belongs to $\mathcal{A N}(H)$. In fact, we show that a bounded operator $T$ defined on an infinite dimensional Hilbert space is hyponormal and belongs to $\mathcal{A N}(H)$ if and only if there exists a unique triple $(K, F, \alpha)$, where $K$ is a positive compact operator, $F$ is a positive finite rank operator, $\alpha$ is positive real number such that $T=K-F+\alpha I$ and $K F=0, F \leq \alpha I$. In fact, here $\alpha=m_{e}(T)$, the essential minimum modulus of $T$. Moreover, we give explicit structure of self-adjoint and $\mathcal{A N}$-operators as well as hyponormal and $\mathcal{A N}$-operators. Finally, we also obtain structure of general $\mathcal{A N}$-operators. In the process we also prove several important properties of $\mathcal{A N}$-operators. Unless otherwise stated, the hyponormal operators in

2010 Mathematics Subject Classification. Primary: 47A75; Secondaries: 47A10 .

Key words and phrases. Norm-attainability ; Compactness; Hyponormal operators.

(C)2020 Proceedings of International Mathematical Sciences.

Submitted on 15-February, 2020 Published on December 30th, 2020. Communicated by Ekrem SAVAS.

The author is thankful to the reviewers for their useful comments . 
this work are compact. We organize the article as follows: Section 1: Introduction; Section 2: Preliminaries and notations; Section 3: Main results.

\section{Preliminaries}

In this section, we give the preliminaries. These include the basic terms, definitions and notations which are useful in the sequel. Throughout the paper, we consider all Hilbert spaces to be infinite dimensional and complex. We denote inner product and the induced norm by by $\langle.,$.$\rangle and \|\cdot\|$ respectively. The unit sphere of a closed subspace $M$ of $H$ is denoted by $S_{M}:=\{x \in M:\|x\|=1\}$ and $P_{M}$ denote the orthogonal projection $P_{M}: H \rightarrow H$ with range $M$. The identity operator on $M$ is denoted by $I_{M}$. See details in [1] and the references therein.

Definition 2.1. An operator $T: H_{1} \rightarrow H_{2}$ is said to be bounded if there exists a $C>0$ such that $\|T x\| \leq C\|x\|$, for all $x \in H_{1}$. If $T$ is bounded, the quantity $\|T\|=\sup \left\{\|T x\|: x \in S_{H_{1}}\right\}$ is finite and is called the norm of $T$.

We denote the space of all bounded linear operators between $H_{1}$ and $H_{2}$ by $\mathcal{B}\left(H_{1}, H_{2}\right)$. In general, the set of all bounded linear operators on $H$ is denoted by $\mathcal{B}(H)$.

Definition 2.2. For $T \in \mathcal{B}\left(H_{1}, H_{2}\right)$, there exists a unique operator denoted by $T^{*}: H_{2} \rightarrow H_{1}$ called the adjoint operator satisfying $\langle T x, y\rangle=\left\langle x, T^{*} y\right\rangle$, for all $x \in$ $H_{1}$ and for all $y \in H_{2}$.

Definition 2.3. An operator $T \in \mathcal{B}\left(H_{1}, H_{2}\right)$ is said to be norm attaining if there exists a $x \in S_{H_{1}}$ such that $\|T x\|=\|T\|$. We denote the class of norm attaining operators by $\mathcal{N}\left(H_{1}, H_{2}\right)$.

Remark. It is known that $\mathcal{N}\left(H_{1}, H_{2}\right)$ is dense in $\mathcal{B}\left(H_{1}, H_{2}\right)$ with respect to the operator norm of $\mathcal{B}\left(H_{1}, H_{2}\right)$. We refer to [2] for more details.

Definition 2.4. (1]). An operator $T \in \mathcal{B}\left(H_{1}, H_{2}\right)$ is said to be absolutely norm attaining or $\mathcal{A N}$-operator (shortly), if $\left.T\right|_{M}$, the restriction of $T$ to $M$, is norm attaining for every non zero closed subspace $M$ of $H_{1}$. That is $\left.T\right|_{M} \in \mathcal{N}\left(M, H_{2}\right)$ for every non zero closed subspace $M$ of $H_{1}$.

Definition 2.5. An operator $T \in \mathcal{B}(H)$ is said to be hyponormal if $\left\|T^{*} x\right\| \leq\|T x\|$, for all $x \in H$.

Remark. This class contains $\mathcal{K}\left(H_{1}, H_{2}\right)$, and the class of partial isometries with finite dimensional null space or finite dimensional range space.

In the remaining part of this section, we give standard terminologies and notations found in 3. Let $T \in \mathcal{B}(H)$. Then $T$ is said to be normal if $T^{*} T=T T^{*}$, self-adjoint if $T=T^{*}$. If $T$ is self-adjoint and $\langle T x, x\rangle \geq 0$, for all $x \in H$, then $T$ is called positive. It is well known that for a positive operator $T$, there exists a unique positive operator $S \in \mathcal{B}(H)$ such that $S^{2}=T$. We write $S=T^{\frac{1}{2}}$ and is called as the positive square root of $T$. If $S, T \in \mathcal{B}(H)$ are self-adjoint and $S-T \geq 0$, then we write this by $S \geq T$. If $P \in \mathcal{B}(H)$ is such that $P^{2}=P$, then $P$ is called a projection. If Null space of $P, N(P)$ and range of $P, R(P)$ are orthogonal to each other, then $P$ is called an orthogonal projection. It is a well known fact that a projection $P$ is an orthogonal projection if and only if it is self-adjoint if and only if it is normal. We call an operator $V \in \mathcal{B}\left(H_{1}, H_{2}\right)$ to 
be an isometry if $\|V x\|=\|x\|$, for each $x \in H_{1}$. An operator $V \in \mathcal{B}\left(H_{1}, H_{2}\right)$ is said to be a partial isometry if $\left.V\right|_{N(V)^{\perp}}$ is an isometry. That is, $\|V x\|=\|x\|$ for all $x \in N(V)^{\perp}$. If $V \in \mathcal{B}(H)$ is isometry and onto, then $V$ is said to be a unitary operator. If $T \in \mathcal{B}(H)$ is a self-adjoint operator, then $T=T_{+}-T_{-}$, where $T_{ \pm}$are positive operators. Here $T_{+}$is called the positive part and $T_{-}$is called the negative part of $T$. Moreover, this decomposition is unique. In general, if $T \in \mathcal{B}\left(H_{1}, H_{2}\right)$, then $T^{*} T \in \mathcal{B}\left(H_{1}\right)$ is positive and $|T|:=\left(T^{*} T\right)^{\frac{1}{2}}$ is called the modulus of $T$. In fact, there exists a unique partial isometry $V \in \mathcal{B}\left(H_{1}, H_{2}\right)$ such that $T=V|T|$ and $N(V)=N(T)$. This factorization is called the polar decomposition of $T$. If $T \in \mathcal{B}(H)$, then $T=\frac{T+T^{*}}{2}+i\left(\frac{T-T^{*}}{2 i}\right)$. The operators $\operatorname{Re}(T):=\frac{T+T^{*}}{2}$ and $\operatorname{Im}(T):=\frac{T-T^{*}}{2 i}$ are self-adjoint and called the real and the imaginary parts of $T$ respectively. A closed subspace $M$ of $H$ is said to be invariant under $T \in \mathcal{B}(H)$ if $T M \subseteq M$ and reducing if both $M$ and $M^{\perp}$ are invariant under $T$. For $T \in \mathcal{B}(H)$, the set $\rho(T):=\left\{\lambda \in \mathbb{C}: T-\lambda I: H \rightarrow H\right.$ is invertible and $\left.(T-\lambda I)^{-1} \in \mathcal{B}(H)\right\}$ is called the resolvent set and the complement $\sigma(T)=\mathbb{C} \backslash \rho(T)$ is called the spectrum of $T$. The spectral radius of $T$ is given by $m(T)=\sup \{|\lambda| \in \mathbb{C}: \lambda \in \rho(T)\}$. It is well known that $\sigma(T)$ is a non empty compact subset of $\mathbb{C}$. The point spectrum of $T$ is defined by $\sigma_{p}(T)=\{\lambda \in \mathbb{C}: T-\lambda I$ is not one-to-one $\}$. Note that $\sigma_{p}(T) \subseteq \sigma(T)$. A self-adjoint operator $T \in \mathcal{B}(H)$ is positive if and only if $\sigma(T) \subseteq[0, \infty)$. If $T \in \mathcal{B}\left(H_{1}, H_{2}\right)$, then $T$ is said to be compact if for every bounded set $S$ of $H_{1}$, the set $T(S)$ is pre-compact in $H_{2}$. Similarly, for every bounded sequence $\left(x_{n}\right)$ of $H_{1}$, $\left(T x_{n}\right)$ has a convergent subsequence in $H_{2}$. We denote the set of all compact operators between $H_{1}$ and $H_{2}$ by $\mathcal{K}\left(H_{1}, H_{2}\right)$. In case if $H_{1}=H_{2}=H$, then $\mathcal{K}\left(H_{1}, H_{2}\right)$ is denoted by $\mathcal{K}(H)$. A bounded linear operator $T: H_{1} \rightarrow H_{2}$ is called finite rank if $R(T)$ is finite dimensional. The space of all finite rank operators between $H_{1}$ and $H_{2}$ is denoted by $\mathcal{F}\left(H_{1}, H_{2}\right)$ and we write $\mathcal{F}(H, H)=\mathcal{F}(H)$. These standard facts can be obtained in [3] and the references therein.

\section{Main Results}

In this section, we give the main results of this work. We begin with the following auxiliary propositions.

Proposition 3.1. Let $T \in \mathcal{B}\left(H_{1}, H_{2}\right)$ be compact and hyponormal. Then

(i). $m(T)=m(|T|)$

(ii). $m(T)=d(0, \sigma(|T|))$

(iii). $m(T)>0$ if and only if $R(T)$ is closed and $T$ is one-to-one ( $T$ is bounded below)

(iv). in Particular if $H_{1}=H_{2}=H$ and $T^{-1} \in \mathcal{B}(H)$, then $m(T)=\frac{1}{\left\|T^{-1}\right\|}$

(v). if $H_{1}=H_{2}=H$ and $T$ is normal, then

(a) $m(T)=d(0, \sigma(T))$

(b) $m(T)=m\left(T^{*}\right)$

(c) $m\left(T^{n}\right)=m(T)^{n}$ for each $n \in \mathbb{N}$

(vi). if $T \geq 0$, then $m(T)=m\left(T^{\frac{1}{2}}\right)^{2}$.

Proof. The proof is analogous to the proof of Carvajal and Neves. See [1] for proof. 
Proposition 3.2. Let $T=K+F+\alpha I$, where $K$ is a positive compact hyponormal operator, $F$ is a self-adjoint finite rank normal operators and $\alpha>0$. Then the following holds:

(i). $R(T)$ is closed

(ii). $N(T)$ is finite dimensional. In fact, $N(T) \subseteq R(F)$

(iii). $T$ is one-to-one if $K \geq F$

(iv). if $T$ is not a finite rank operator, there exists $a>0, b>0$ such that $\alpha \in(a \gamma(T), b \gamma(T))$

(v). if $T$ is a finite rank operator, then $H$ is finite dimensional.

Proof. By proposition 3.2 above and analogous to the proof in [1] the proof is complete.

Proposition 3.3. Let $T \in \mathcal{B}(\mathcal{H})$ be compact and hyponormal and $\beta \in W_{e}(S)$ where $\alpha>0$. Then there exists an operator $S \in \mathcal{B}(\mathcal{H})$ such that $\|S\|=\|Z\|$, $\|S-Z\|<\alpha$ and $T$ is absolutely norm attaining. Furthermore, there exists a vector $\eta \in H,\|\eta\|=1$ such that $\|Z \eta\|=\|Z\|$ with $\langle Z \eta, \eta\rangle=\beta$.

Proof. Consider $S \in \mathcal{B}(\mathcal{H})$ to be contractive then we may assume that $\|S\|=1$ by ignoring the strict inequality. and also that $0<\alpha<2$. Let $x_{n} \in H(n=$ $1,2, \ldots)$ be such that $\left\|x_{n}\right\|=1,\left\|S x_{n}\right\| \rightarrow 1$ and also $\lim _{n \rightarrow \infty}\left\langle S x_{n}, x_{n}\right\rangle=\beta$. Let $S=G L$ be the polar decomposition of $S$. Here $G$ is a partial isometry and we write $L=\int_{0}^{1} \beta d E_{\beta}$, the spectral decomposition of $L=\left(S^{*} S\right)^{\frac{1}{2}}$. Since $\lim _{n \rightarrow \infty}\left\|S x_{n}\right\|=$ $\|S\|=\|L\|=1$, we have that $\left\|L x_{n}\right\| \rightarrow 1$ as $n$ tends to $\infty$ and $\lim _{n \rightarrow \infty}\left\langle S x_{n}, x_{n}\right\rangle=$ $\lim _{n \rightarrow \infty}\left\langle G L x_{n}, x_{n}\right\rangle=\lim _{n \rightarrow \infty}\left\langle L x_{n}, G^{*} x_{n}\right\rangle$. Now for $H=\overline{R(L)} \oplus \operatorname{Ker} L$, we can choose $x_{n}$ such that $x_{n} \in \overline{R(L)}$ for large $n$. Indeed, let $x_{n}=x_{n}^{(1)} \oplus x_{n}^{(2)}, n=1,2, \ldots$ Then we have that $L x_{n}=L x_{n}^{(1)} \oplus L x_{n}^{(2)}=L x_{n}^{(1)}$ and that $\lim _{n \rightarrow \infty}\left\|x_{n}^{(1)}\right\|=$ 1 , $\lim _{n \rightarrow \infty}\left\|x_{n}^{(2)}\right\|=0$ since $\lim _{n \rightarrow \infty}\left\|L x_{n}\right\|=1$. Replacing $x_{n}$ with $\frac{x_{n}^{(1)}}{\left\|x_{n}^{(1)}\right\|}$, we obtain $\lim _{n \rightarrow \infty}\left\|L \frac{1}{\left\|x_{n}^{(1)}\right\|} x_{n}^{(1)}\right\|=\lim _{n \rightarrow \infty}\left\|S \frac{1}{\left\|x_{n}^{(1)}\right\|} x_{n}^{(1)}\right\|=1$, and we also obtain that $\lim _{n \rightarrow \infty}\left\langle S \frac{1}{\left\|x_{n}^{(1)}\right\|} x_{n}^{(1)}, \frac{1}{\left\|x_{n}^{(1)}\right\|} x_{n}^{(1)}\right\rangle=\beta$. Now assume that $x_{n} \in \overline{R L}$. Since $G$ is a partial isometry from $\overline{R(L)}$ onto $\overline{R(S)}$, we have that $\left\|G x_{n}\right\|=1$ and $\lim _{n \rightarrow \infty}\left\langle L x_{n}, G^{*} x_{n}\right\rangle=\beta$. Since $L$ is a positive operator, $\|L\|=1$ and for any $x \in H$, $\langle L x, x\rangle \leq\langle x, x\rangle=\|x\|^{2}$. Replacing $x$ with $L^{\frac{1}{2}} x$, we get that $\left\langle L^{2} x, x\right\rangle \leq\langle L x, x\rangle$, where $L^{\frac{1}{2}}$ is the positive square root of $L$. Therefore we have that $\|L x\|^{2}=$ $\langle L x, L x\rangle \leq\langle L x, x\rangle$. It is obvious that $\lim _{n \rightarrow \infty}\left\|L x_{n}\right\|=1$ and that $\left\|L x_{n}\right\|^{2} \leq$ $\left\langle L x_{n}, x_{n}\right\rangle \leq\left\|L x_{n}\right\|^{2}=1$. Hence, $\lim _{n \rightarrow \infty}\left\langle L x_{n}, x_{n}\right\rangle=1=\|L\|$. Moreover, Since $I-L \geq 0$, we have $\lim _{n \rightarrow \infty}\left\langle(I-L) x_{n}, x_{n}\right\rangle=0$. thus $\lim _{n \rightarrow \infty}\left\|(I-L)^{\frac{1}{2}} x_{n}\right\|=0$.

Indeed, $\lim _{n \rightarrow \infty}\left\|(I-L) x_{n}\right\| \leq \lim _{n \rightarrow \infty}\left\|(I-L)^{\frac{1}{2}}\right\| \cdot\left\|(I-L)^{\frac{1}{2}} x_{n}\right\|=0$. For $\alpha>0$, let $\gamma=\left[0,1-\frac{\alpha}{2}\right]$ and let $\rho=\left(1-\frac{\alpha}{2}, 1\right]$. We have $L=\int_{\gamma} \mu d E_{\mu}+\int_{\rho} \mu d E_{\mu}=$ $L E(\gamma) \oplus L E(\rho)$. Next we show that $\lim _{n \rightarrow \infty}\left\|E(\gamma) x_{n}\right\|=0$. If there exists a subsequence $x_{n_{i}},(i=1,2, \ldots$,$) such that \left\|E(\gamma) x_{n_{i}}\right\| \geq \epsilon>0,(i=1,2, \ldots$,$) , then$ since $\lim _{i \rightarrow \infty}\left\|x_{n_{i}}-L x_{n_{i}}\right\|=0$, it follows from [4 that $\lim _{i \rightarrow \infty}\left\|x_{n_{i}}-L x_{n_{i}}\right\|^{2}=$ $\lim _{i \rightarrow \infty}\left(\left\|E(\gamma) x_{n_{i}}-L E(\gamma) x_{n_{i}}\right\|^{2}+\left\|E(\rho) x_{n_{i}}-L E(\rho) x_{n_{i}}\right\|^{2}\right)=0$. Hence we have that $\lim _{i \rightarrow \infty}\left\|E(\gamma) x_{n_{i}}-L E(\gamma) x_{n_{i}}\right\|^{2}=0$. Now it is clear that $\left\|E(\gamma) x_{n_{i}}-L E(\gamma) x_{n_{i}}\right\| \geq$ $\left.\left\|E(\gamma) x_{n_{i}}\right\|-\|L E(\gamma)\| . \| E \gamma\right) x_{n_{i}}\|\geq(I-\|L E(\gamma)\|)\| E(\gamma) x_{n_{i}} \| \geq \frac{\alpha}{2} \epsilon>0$. This is a contradiction. Therefore, $\lim _{n \rightarrow \infty}\left\|E(\gamma) x_{n}\right\|=0$. Since $\lim _{n \rightarrow \infty}\left\langle L x_{n}, x_{n}\right\rangle=1$, we have that $\lim _{n \rightarrow \infty}\left\langle L E(\rho) x_{n}, E(\rho) x_{n}\right\rangle=1$ and $\lim _{n \rightarrow \infty}\left\langle E(\rho) x_{n}, G^{*} E(\rho) x_{n}\right\rangle=\beta$. It 
is easy to see that $\lim _{n \rightarrow \infty}\left\|E(\rho) x_{n}\right\|=1, \lim _{n \rightarrow \infty}\left(L \frac{E(\rho) x_{n}}{\left\|E(\rho) x_{n}\right\|}, \frac{E(\rho) x_{n}}{\left\|E(\rho) x_{n}\right\|}\right)=1$ and $\lim _{n \rightarrow \infty}\left(L \frac{E(\rho) x_{n}}{\left\|E(\rho) x_{n}\right\|}, G^{*} \frac{E(\rho) x_{n}}{\left\|E(\rho) x_{n}\right\|}\right)=\beta$ Replacing $x$ with $\frac{E(\rho) x_{n}}{\left\|E(\rho) x_{n}\right\|}$, we can assume that $x_{n} \in E(\rho) H$ for each $n$ and $\left\|x_{n}\right\|=1$. Let $J=\int_{\gamma} \mu d E_{\mu}+\int_{\rho} \mu d E_{\mu}=J_{1} \oplus E(\rho)$. Then it is evident that $\|J\|=\|S\|=\|L\|=1, J x_{n}=x_{n}$ and $\|J-L\| \leq \frac{\alpha}{2}$. If we can find a contraction $V$ such that $V-G \leq \frac{\alpha}{2}$ and $\left\|V x_{n}\right\|=1$ and $\left\langle V x_{n}, x_{n}\right\rangle=\beta$, for a large $n$ then letting $Z=V J$, we have that $\left\|Z x_{n}\right\|=\left\|V J x_{n}\right\|=1$, and that $\left\langle Z x_{n}, x_{n}\right\rangle=\left\langle V J x_{n}, x_{n}\right\rangle=\left\langle V x_{n}, x_{n}\right\rangle=\beta\|S-Z\|=\|G L-V J\|=\alpha$. To complete the proof, we now construct the desired contraction $V$. Clearly, $\lim _{n \rightarrow \infty}\left\langle x_{n}, G^{*} x_{n}\right\rangle=\beta$, because $\lim _{n \rightarrow \infty}\left\langle L x_{n}, G^{*} x_{n}\right\rangle=\beta$ and $\lim _{n \rightarrow \infty} \| x_{n}-$ $L x_{n} \|=0$. Let $G x_{n}=\phi_{n} x_{n}+\varphi_{n} y_{n}, \quad\left(y_{n} \perp x_{n},\left\|y_{n}\right\|=1\right)$ then $\lim _{n \rightarrow \infty} \phi_{n}=\beta$, because $\lim _{n \rightarrow \infty}\left\langle G x_{n}, x_{n}\right\rangle=\lim _{n \rightarrow \infty}\left\langle x_{n}, G^{*} x_{n}\right\rangle=\beta$ but $\left\|G x_{n}\right\|^{2}=\left|\phi_{n}\right|^{2}+\left|\varphi_{n}\right|^{2}=1$, so we have that $\lim _{n \rightarrow \infty}\left|\varphi_{n}\right|=\left.\sqrt{1-\mid \beta}\right|^{2}$. Then by [4] and [5] the remaining part of the proof is analogous and this completes the proof.

At this point, we consider absolute norm-attainability for commutators of compact hypomormal operators.

Lemma 3.4. Let $E \in \mathcal{B}(H)$ be compact hyponormal then $E X-X E$ is absolutely norm attaining if there exists a vector $\zeta \in H$ such that $\|\zeta\|=1, \quad\|E \zeta\|=$ $\|E\|,\langle E \zeta, \zeta\rangle=0$.

Proof. Let $x \in H$ satisfy $x \perp\{\zeta, E \zeta\}$, and define a compact $X$ as follows $X: \zeta \rightarrow$ $\zeta, \quad E \zeta \rightarrow-E \zeta, \quad x \rightarrow 0$. Since $X$ is a bounded operator on $H$ and $\|X \zeta\|=$ $\|X\|=1,\|E X \zeta-X E \zeta\|=\|E \zeta-(-E \zeta)\|=2\|E \zeta\|=2\|E\|$. It follows that $\|E X-X E\|=2\|E\|$ by Proposition 3.1 , because $\langle E \zeta, \zeta\rangle=0 \in W_{e}(E)$. Hence we have that $\|E X-X E\|=2\|E\|$. Therefore, $E X-X E$ is absolutely norm attaining.

Lemma 3.5. Let $S, T \in \mathcal{B}(H)$ be compact hyponormal. If there exists vectors $\zeta, \eta \in H$ such that $\|\zeta\|=\|\eta\|=1, \quad\|S \zeta\|=\|S\|,\|T \eta\|=\|T\|$ and $\frac{1}{\|S\|}\langle S \zeta, \zeta\rangle=$ $-\frac{1}{\|T\|}\langle T \eta, \eta\rangle$, then $S X-X T$ is is absolutely norm attaining.

Proof. Since $H$ has an orthonormal basis then by linear dependence of vectors, if $\eta$ and $T \eta$ are linearly dependent, i.e.,T $T=\phi\|T\| \eta$, then we have $|\phi|=1$ and $|\langle T \eta, \eta\rangle|=\|T\|$. It follows that $|\langle S \zeta, \zeta\rangle|=\|S\|$ which implies that $S \zeta=\varphi\|S\| \zeta$ and $|\varphi|=1$. Hence $\left\langle\frac{S \zeta}{\|S\|}, \zeta\right\rangle=\varphi=-\left\langle\frac{T \eta}{\|T\|}, \eta\right\rangle=-\phi$. Defining $X$ as $X: \eta \rightarrow$ $\zeta, \quad\{\eta\}^{\perp} \rightarrow 0$, we have $\|X\|=1$ and $(S X-X T) \eta=\varphi(\|S\|+\|T\|) \zeta$, which implies that $\|S X-X T\|=\|(S X-X T) \eta\|=\|S\|+\|T\|$. By 2, it follows that $\|S X-X T\|=\|S\|+\|T\|=\left\|\delta_{S, T}\right\|$. That is $S X-X T$ is absolutely norm attaining. If $\eta$ and $T \eta$ are linearly independent, then $\left|\left\langle\frac{T \eta}{\|T\|}, \eta\right\rangle\right|<1$, which implies that $\left|\left\langle\frac{S \zeta}{\|S\|}, \zeta\right\rangle\right|<1$. Hence $\zeta$ and $S \zeta$ are also linearly independent. Let us redefine $X$ as follows: $X: \eta \rightarrow \zeta, \quad \frac{T \eta}{\|T\|} \rightarrow-\frac{S \zeta}{\|S\|}, \quad x \rightarrow 0$, where $x \in\{\eta, T \eta\}^{\perp}$. We show that $X$ is a partial isometry. Let $\frac{T \eta}{\|T\|}=\left\langle\frac{T \eta}{\|T\|}, \eta\right\rangle \eta+\tau h, \quad\|h\|=1, h \perp \eta$. Since $\eta$ and $T \eta$ are linearly independent, $\tau \neq 0$. So we have that $X \frac{T \eta}{\|T\|}=\left\langle\frac{T \eta}{\|T\|}, \eta\right\rangle X \eta+\tau X h=$ $-\left\langle\frac{S \zeta}{\|S\|}, \zeta\right\rangle \zeta+\tau X h$, which implies that $\left\langle X \frac{T \eta}{\|T\|}, \zeta\right\rangle=-\left\langle\frac{S \zeta}{\|S\|}, \zeta\right\rangle+\tau\langle X h, \zeta\rangle=$ 
$-\left\langle\frac{S \zeta}{\|S\|}, \zeta\right\rangle$. It follows then that $\langle X h, \zeta\rangle=0$ i.e., $X h \perp \zeta(\zeta=X \eta)$. Hence we have that $\left\|\left\langle\frac{S \zeta}{\|S\|}, \zeta\right\rangle \zeta\right\|^{2}+\|\tau X h\|^{2}=\left\|X \frac{T \eta}{\|T\|}\right\|^{2}=\left|\left\langle\frac{T \eta}{\|T\|}, \eta\right\rangle\right|^{2}+|\tau|^{2}=1$, which implies that $\|X h\|=1$. Now it is evident that $X$ a partial isometry and $\|(S X-X T) \zeta\|=$ $\|S X-X T\|=\|S\|+\|T\|$, which is equivalent to $\left\|\delta_{S, T}(X)\right\|=\|S\|+\|T\|$. By Lemma 3.1 and [4, $\|S X-X T\|=\|S\|+\|T\|$. Hence $S X-X T$ is absolutely norm attaining.

Corollary 3.6. Let $S, T \in \mathcal{B}(H)$ If both $S$ and $T$ are absolutely norm attaining then the operator $S X T$ is also absolutely norm attaining.

Proof. We can assume that $\|S\|=\|T\|=1$. If both $S$ and $T$ are absolutely norm attaining, then there exists unit vectors $\zeta$ and $\eta$ with $\|S \zeta\|=\|T \eta\|=1$. We can therefore define an operator $X$ by $X=\langle\cdot, T \eta\rangle \zeta$. Clearly, $\|X\|=1$. Therefore, we have $\|S X T\| \geq\|S X T \eta\|=\|\| T \eta\left\|^{2} S \zeta\right\|=1$. Hence, $\|S X T\|=1$, that is $S X T$ is also absolutely norm attaining.

Proposition 3.7. Let $T \in \mathcal{A N}(H)$ be a self-adjoint compact hyponormal operator. Then there exists an orthonormal basis consisting of eigenvectors of $T$.

Proof. The proof follows in the analogously as in 1 but we include it for completeness. Let $\mathcal{B}=\left\{x_{\alpha}: \alpha \in I\right\}$ be the maximal set of orthonormal eigenvectors of $T$. This set is non empty, as $T=T^{*} \in \mathcal{A N}(H)$. Let $M=\overline{\operatorname{span}}\left\{x_{\alpha}: \alpha \in I\right\}$. Then we claim that $M=H$. If not, $M^{\perp}$ is a proper non-zero closed subspace of $H$ and it is invariant under $T$. Since $T=T^{*} \in \mathcal{A N}(H)$, then we have either $\left\|T \mid M^{\perp}\right\|$ or -||$T\left|M^{\perp}\right| \mid$ is an eigenvalue for $T \mid M^{\perp}$. Hence there is a non-zero vector, say $x_{0}$ in $M^{\perp}$, such that $T x_{0}= \pm \| T\left|M^{\perp}\right| \mid x_{0}$. Since $M \cap M^{\perp}=\{0\}$, we have arrived to a contradiction to the maximality of $\mathcal{B}$.

Next, we need to do a characterization for self-adjoint hyponormal compact operators. We ask the following question: For a compact hyponormal self-adjoint operator, can we find $\alpha \in \mathbb{R}$ such that $K+\alpha I \in \mathcal{A N}(H)$. To solve this first we need to answer the question when $K+\alpha I \in \mathcal{N}(H)$. Here we have the following characterization.

Lemma 3.8. Let $K \in \mathcal{K}(H)$ be self-adjoint and $a \in \mathbb{R}$. Let $K=\operatorname{diag}\left(\lambda_{1}, \lambda_{2}, \lambda_{3}, \ldots,\right)$ with respect to orthonormal basis of $H$. Then the following are equivalent:

(i). $T \in \mathcal{N}(H)$

(ii). there exists $n_{0} \in \mathbb{N}$ such that $\left|\lambda_{n_{0}}+a\right|>|a|$.

Proof. The proof is trivial.

Consider $T=T^{*} \in \mathcal{B}(H)$ and have the polar decomposition $T=V|T|$. Let $H_{0}=N(T), H_{+}=N(I-V)$ and $H_{-}=N(I+V)$. Then $H=H_{0} \oplus H_{+} \oplus H_{-}$ which are all invariant under $T$. Let $T_{0}=\left.T\right|_{N(T)}, T_{+}=\left.T\right|_{H_{+}}$and $T_{-}=\left.T\right|_{H_{-}}$. Then $T=T_{0} \oplus T_{+} \oplus T_{-}$. Further more, $T+$ is strictly positive, $T_{-}$is strictly negative and $T_{0}=0$ if $N(T) \neq\{0\}$. Let $P_{0}=P_{N(T)}, P_{ \pm}=P_{H_{ \pm}}$. Then $P_{0}=I-V^{2}$ and $P_{ \pm}=\frac{1}{2}\left(V^{2} \pm V\right)$. Thus $V=P_{+}-P_{-}$. For details see [3].

Theorem 3.9. Let $T \in \mathcal{A N}(H)$ be compact hyponrmal and self-adjoint with the polar decomposition $T=V|T|$. Then the operator $T$ can be represented as $T=$ 
$K-F+\alpha V$, where $K \in \mathcal{K}(H), F \in \mathcal{F}(H)$ are self-adjoint with $K F=0$ and $F^{2} \leq \alpha^{2} I$

Proof. Let $H=H_{+} \oplus H_{-}$and $T=T_{+} \oplus T_{-}$. Since $H_{ \pm}$reduces $T$, we have $T_{ \pm} \in \mathcal{B}\left(H_{ \pm}\right)$. As $T \in \mathcal{A N}(H)$, we have that $T_{ \pm} \in \mathcal{A N}\left(H_{ \pm}\right)$. Hence by [2], we have that $T_{+}=K_{+}-F_{+}+\alpha I_{H_{+}}$such that $K_{+}$is positive compact operator, $F_{+}$ is finite rank positive operator with the property that $K_{+} F_{+}=0$ and $F_{+} \leq \alpha I_{H_{+}}$. As $T_{+}$is strictly positive, $\alpha>0$. Similarly, $T_{-} \in \mathcal{A N}\left(H_{-}\right)$and strictly negative. Hence there exists a triple $\left(K_{-}, F_{-}, \beta\right)$ such that $-T_{-}=K_{-}-F_{-}+\beta I_{H_{-}}$, where $K_{-} \in \mathcal{K}\left(H_{-}\right)$is positive, $F_{-} \in \mathcal{F}\left(H_{-}\right)$is positive with $K_{-} F_{-}=0, F_{-} \leq \beta I_{H_{-}}$ and $\beta>0$. The rest follows from [1] and the proof is complete.

Theorem 3.10. A compact self adjoint hyponormal operator $T \in \mathcal{A N}(H)$ has a countable spectrum.

Proof. Since $T=T_{+} \oplus T_{-} \oplus T_{0}$ and all these operators $T_{+}, T_{-}$and $T_{0}$ are $\mathcal{A N}$ operators. We know that $\sigma\left(T_{+}\right), \sigma\left(T_{0}\right)$ are countable, as they are positive. Also, $-T_{-}$is positive $\mathcal{A N}$-operator and hence $\sigma\left(T_{-}\right)$is countable. Hence we can conclude that $\sigma(T)=\sigma\left(T_{+}\right) \cup \sigma\left(T_{-}\right) \cup \sigma\left(T_{0}\right)$ is countable.

Now we consider the structure of normal $\mathcal{A N}$-operators. We see this in the next lemma

Lemma 3.11. Let $T \in \mathcal{A N}(H)$ be compact hyponormal with the polar decomposition $T=V|T|$. Then there exists a compact hyponormal operator $K$, a finite rank normal operator $F \in \mathcal{B}(H)$ such that $V, K, F$ are mutually commutative.

Proof. We have $V K=V V K_{1}=V K_{1} V=K V$ and $V F=V V F_{1}=V F_{1} V=F V$. Also, $K F=0=F K$.

Theorem 3.12. Let $T \in \mathcal{B}(H)$ be compact hyponormal. Then $T \in \mathcal{A N}(H)$ if and only if $T^{*} \in \mathcal{A N}(H)$.

Proof. We know that $T \in \mathcal{A N}(H)$ if and only if $T^{*} T \in \mathcal{A N}(H)$. Since $T^{*} T=$ $T T^{*}$, by Corollary Lemma 3.4 again, it follows that $T T^{*} \in \mathcal{A N}(H)$ if and only if $T^{*} \in \mathcal{A N}(H)$.

Acknowledgement. This work was partially supported financially by the DFG Grant No. 1603991000.

\section{REFERENCES}

[1] X. Carvajal and W. Neves, Operators that achieve the norm, I. Eq. Oper. Theory, 72(2)(2012), 179-195.

[2] P. Enflo, J. Kover and L. Smithies, Denseness for norm attaining operator-valued functions, Linear Algebra Appl., 338(2001), 139-144.

[3] P. R. Halmos, A Hilbert space problem book, Springer-Verlag, New York, 1982.

[4] N. B. Okelo, The norm attainability of some elementary operators, Appl. Math. E-Notes, 13(2013), 1-7.

[5] G. Ramesh, Absolutely Norm attaining Paranormal operators, J. Math. Anal. Applic., 465(1)(2018), 547-556.

Benard OKelo,

Department of Pure and Applied Mathematics, Jaramogi Oginga, Odinga University of Science and Technology, P. O. Box 210-40601, Bondo, Kenya

ORCID NUMBER:0000-0003-3963-1910

Email address: bnyaare@yahoo.com 